\title{
DISCRETE ANALYTIC FUNCTIONS OF EXPONENTIAL GROWTH
}

\author{
BY \\ DORON ZEILBERGER
}

\begin{abstract}
Analogues of classical representation formulas for entire functions of exponential type are proved in the class of discrete analytic functions.
\end{abstract}

1. Introduction. Let $Z$ be the group of integers, and consider the class of functions $f: Z \times Z \rightarrow C$ for which

$$
\begin{aligned}
L f(m, n) \equiv & f(m, n)+i f(m+1, n) \\
& -f(m+1, n+1)-i f(m, n+1)=0
\end{aligned}
$$

for every $(m, n) \in Z \times Z$.

Such functions, termed discrete entire, were first considered by FerrandLelong [1] and their theory was developed by Duffin [2] and others.

In this paper we shall prove theorems on discrete analytic functions of exponential growth which are analogous to certain classical theorems about entire functions of exponential type (Boas [3] is the standard reference for the latter). Perhaps the main result of this paper is a proof of the discrete analogue of the (two-sided) Paley-Wiener theorem (Theorem 3.4). Our methods, which are completely different from the ones used in the classical theory, use duality arguments on certain Banach spaces of analytic functions of two complex variables. In essence the trick is to translate into discrete language a "continuous" idea due to Ehrenpreis [4] (see the preface of the latter). Ehrenpreis deals with the solutions of partial differential equations, whereas present interest focuses on solutions of the simple partial difference equation $L f=0$, based upon the Duffin operator $L$ introduced in (1.1). Since the discrete case is, by its nature, simpler than the continuous one, no explicit reference need be made to Ehrenpreis [4].

Received by the editors November $10,1975$.

AMS (MOS) subject classifications (1970). Primary 30A95; Secondary 30A98, 30A64.

Key words and phrases. Discrete analytic functions, entire function of exponential type, PaleyWiener theorem. 
The key idea of this paper is to assciate with each discrete function $f: Z \times Z \rightarrow C$ a linear functional $T_{f}$ defined on the algebra

$$
\mathbb{Q}=\left\{\sum_{m=-M}^{M} \sum_{n=-N}^{N} a_{m n} z^{m} w^{n} ; a_{m n} \in \mathrm{C}, M, N \text { integers }\right\}
$$

of polynomials in $z, z^{-1}, w, w^{-1}$ which is given by

$$
T_{f}\left(\sum_{-M}^{M} \sum_{-N}^{N} a_{m n} z^{m} w^{n}\right)=\sum_{-M}^{M} \sum_{-N}^{N} a_{m n} f(m, n)
$$

and using the fact that (1.1) holds iff

$$
T_{f}\left((1+i z-z w-i w) z^{m} w^{m}\right)=0 \quad \forall(m, n) \in Z \times Z,
$$

we get that $f(m, n)$ is discrete entire iff $T_{f}$ annihilates $(1+i z-z w-i w)$, the ideal.

We shall first consider, in \$2, discrete analytic functions of exponential growth defined only on the upper right quarter lattice $Z^{+} \times Z^{+}=\{(m, n) ; m$ $\geqslant 0, n \geqslant 0\}$.

2. Discrete analytic functions of exponential growth on the upper right quarter lattice. Let $\mathbb{Q}_{+}$be the algebra of polynomials

$$
\left\{\sum_{m=0}^{M} \sum_{n=0}^{N} a_{m, n} z^{m} w^{n} ; a_{m n} \in \mathbf{C}, M, N \text { integers }\right\} .
$$

Any discrete function $f: Z^{+} \times Z^{+} \rightarrow \mathrm{C}$ induces a linear functional $T_{f}$ on $\mathbb{Q}_{+}$ given by

$$
T_{f}\left(\sum_{m=0}^{M} \sum_{n=0}^{N} a_{m n} z^{m} w^{n}\right)=\sum_{m=0}^{M} \sum_{n=0}^{N} a_{m n} f(m, n),
$$

and for any linear functional $T$ on $Q_{+}, T=T_{g}$ where $g(m, n)=T\left(z^{m} w^{n}\right)$.

Let $r, s$ be any positive numbers and consider the polydisc $\{|z|<r\}$ $\times\{|w|<s\}$ in $\mathbf{C}^{2}$. Let $H(r, s)$ be the class of functions holomorphic on this polydisc and continuous on its closure. This is a reflexive Banach space with norm

$$
\|u\|_{\infty}=\sup _{|z|<r ;|w|<s}|u(z, w)|
$$

(see Rudin [5, p. 3]).

Evidently $\mathbb{Q}_{+} \subset H(r, s)$ and, in fact, $\mathbb{Q}_{+}$is dense in $H(r, s)$. We now make the following

Definition. A discrete function $f: Z^{+} \times Z^{+} \rightarrow \mathrm{C}$ is said to be of exponential growth $(R, S)$ if there exists a constant $C$ such that $|f(m, n)|$ 
$\leqslant C R^{m} S^{n}$ for every $(m, n) \in Z^{+} \times Z^{+}$.

Later we shall need the following

LEMMA 2.1. Let $F: Z^{+} \times Z^{+} \rightarrow \mathrm{C}$ be of exponential growth $(R, S)$ and let $r>R, s>S$; then $T_{f}$ defined on $\mathbb{Q}_{+}$by $(2.1)$, can be extended continuously to the Banach space $H(r, s)$.

Proof.

$$
F(z, w)=\sum_{m=0}^{\infty} \sum_{n=0}^{\infty} f(m, n) z^{-(m+1)} w^{-(n+1)}
$$

is defined and holomorphic in $\{|z|>R\} \times\{|w|>S\}$. Let $u(z, w) \in \mathbb{Q}_{+}$; then

$$
T_{f}(u)=\frac{1}{(2 \pi i)^{2}} \int_{\Gamma} F(z, w) u(z, w) d z d w
$$

for some polycontour $\Gamma$ in the polyannulus $\{R<|z|<r\} \times\{S<|w|<s\}$. Thus

$$
\left|T_{f}(u)\right| \leqslant C(F)\|u\|_{\infty}
$$

for some constant $C(F)$ depending only on $F$ (and hence on $f$ ). Since $\mathbb{Q}_{+}$is dense in $H(r, s)$, the lemma is proved.

The "typical" discrete function of exponential growth $(R, S)$ is $f(m, n)$ $=z_{0}^{m} w_{0}^{n}$ for some complex constants $z_{0}, w_{0}$ for which $\left|z_{0}\right|=R,\left|w_{0}\right|=S$ and the induced linear functional $T_{f}$ is the "point evaluator" at $\left(z_{0}, w_{0}\right), J_{\left(z_{0}, w_{0}\right)}$, $T_{f}(u)=u\left(z_{0}, w_{0}\right)=J_{\left(z_{0}, w_{0}\right)}(u)$. If we require that $z_{0}^{m} w_{0}^{n}$ be discrete analytic then $w_{0}=\left(1+i z_{0}\right) /\left(z_{0}+i\right)$ and so the "typical" discrete analytic function of exponential growth is

$$
e(z ; m+i n)=z^{m}((1+i z) /(z+i))^{n}
$$

which is of exponential growth $(|z|,|(z-i) /(z+i)|)$.

The next theorem tells us that every discrete analytic function of exponential growth is in some sense a "linear combination" of (discrete) exponentials $e(z ; m+i n)$.

THEOREM 2.2. Let $f$ be discrete analytic in the quarter lattice $Z^{+} \times Z^{+}$and let it be of exponential growth $(R, S)$ there. Then there exists a plane measure $d \mu(z)$ supported in the region

$$
A_{R, S}=\{z \in \mathbf{C} ;|z| \leqslant R,|(z-i) /(z+i)| \leqslant S\},
$$

for which 


$$
f(m, n)=\int e(z ; m+i n) d \mu(z)
$$

Proof. We proceed by steps.

Step 1. $T_{f}$ annihilates the principal ideal $(1+i z-z w-i w) \mathbb{Q}_{+}$. Proof. Since $f(m, n)$ is discrete analytic in $Z^{+} \times Z^{+}$,

$$
\begin{aligned}
T_{f}\left((1+i z-z w-i w) z^{m} w^{n}\right)= & f(m, n)+i f(m+1, n) \\
& -f(m+1, n+1)-i f(m, n+1) \\
= & L f(m, n)=0
\end{aligned}
$$

for every $(m, n) \in Z^{+} \times Z^{+}$.

Step 2. $T_{f}$ extended to $H(r, s)$ as in Lemma 2.1, annihilates the ideal $(1+i z-z w-i w) H(r, s)$.

Proof. This follows immediately from Step 1 and the fact that $\mathbb{Q}_{+}$is dense in $H(r, s)$.

Step 3. Let

$$
V_{r, s}=\{(z, w) ;|z|<r,|w|<s, 1+i z-z w-i w=0\} ;
$$

then

$$
(1+i z-z w-i w) H(r, s)=\left\{u \in H(r, s) ;\left.u\right|_{r_{r, s}}=0\right\} .
$$

Proof. This is the famous Hilbert semilocal nullstellensatz for a very special case. Suppose $\left.u\right|_{V, s} \equiv 0$; then $v(z, w)=u(z, w) /(1+i z-z w-i w)$ is holomorphic in $\{|z|<r\} \times\{|w|<s\} \sim V_{r, s}$ and locally bounded in $\{|z|<r\} \times\{|w|$ $<s\}$ (Gunning and Rossi [9, p. 19]). By the Riemann Removable Singularity Theorem, $v(z, w)$ can be extended to be holomorphic in $\{|z|<r\} \times\{|w|<s\}$ and is evidently continuous on its closure, i.e., $v(z, w) \in H(r, s)$. Thus

$$
u(z, w)=(1+i z-z w-i w) v(z, w) \in(1+i z-z w-i w) H(r, s) .
$$

The opposite inclusion is trivial.

Step 4. There exists a measure $d \tilde{\mu}(z, w)$ on $C^{2}$ supported in $V_{r, s}$ such that

$$
T_{f}(u)=\int_{V, s} u(z, w) d \tilde{\mu}(z, w), \quad u \in H(r, s) .
$$

Proof. Let $(z, w) \in\{|z|<r\} \times\{|w|<s\}$ and denote by $J_{(z, w)}$ the point evaluator at $(z, w)$ :

$$
J_{(z, w)}(u)=u(z, w)
$$

By Steps 3 and 2 


$$
J_{(z, w)}(u)=0 \quad \forall(z, w) \in V_{r, s} \Rightarrow T_{f}(u)=0 .
$$

Thus the annihilator of $\operatorname{span}\left\{J_{(z, w)} ;(z, w) \in V_{r, s}\right\}$ is contained in the annihilator of $T_{f}$ Since $H(r, s)$ is a reflexive Banach space, it follows (cf. Taylor [6, pp. 225-226]) that $T_{f}$ is contained in the closed linear span of $\left\{\mathrm{J}_{(z, w)} ;(z, w)\right.$ $\left.\in V_{r, s}\right\}$. Consequently there exists a sequence of atomic measures $\left\{d \mu_{n}\right\}$, supported in $V_{r, s}$ such that for every $u \in H(r, s), \int u(z, w) d \mu_{n}(z, w) \rightarrow T_{f}(u)$. By Helly's selection principle there is a measure $d \tilde{\mu}(z, w)$, supported in $V_{r, s}$ such that

$$
\int u(z, w) d \mu_{n} \rightarrow \int u(z, w) d \tilde{\mu} \quad \forall u \in H(r, s),
$$

and we have

$$
T_{f}(u)=\int_{V_{r, s}} u(z, w) d \tilde{\mu}(z, w) .
$$

Step 5. This completes the proof of the theorem. Let $d \mu(z)$ be the "projection" of $d \tilde{\mu}(z, w)$ on C:

$$
\int_{C} v(z) d \mu(z)=\int v(z) d \tilde{\mu}(z, w) .
$$

$d \mu(z)$ is supported in

$$
A_{r, s}^{0}=\{z \in \mathbf{C} ;|z|<r,|(z-i) /(z+i)|<s\}
$$

for every $r>R, s>S$ and, hence, in

$$
A_{R, S}=\{z \in \mathbf{C} ;|z| \leqslant R,|(z-i) /(z+i)| \leqslant S\},
$$

and finally

$$
\begin{aligned}
f(m, n) & =T_{f}\left(z^{m} w^{n}\right)=\int_{V_{r, S}} z^{m} w^{n} d \ddot{\mu}(z, w) \\
& =\int_{A_{R, S}} z^{m}\left(\frac{1+i z}{z+i}\right)^{n} d \mu(z)=\int_{A_{R, S}} e(z ; m+i n) d \mu(z) .
\end{aligned}
$$

Obviously, the knowledge of $\{f(m, 0)\}_{m=0}^{\infty}$ and $\{f(0, n)\}_{n=0}^{\infty}$ uniquely determines the discrete analytic function $f(m, n)$ on the whole of $Z^{+} \times Z^{+}$. The next theorem shows that if $f$ satisfies an appropriate growth condition then the knowledge of $f$ just on the $m$-axis, i.e., the sequence $\{f(m, 0)\}_{m=0}^{\infty}$, determines $f$ on all $Z^{+} \times Z^{+}$.

THEOREM 2.3. If $f$ is discrete analytic on $Z^{+} \times Z^{+}$and of exponential growth $(R, S)$ where $R>1, S<|(R+1) /(R-1)|$, then the values $\{f(m, 0)\}_{m=0}^{\infty}$ uniquely determine $f$. 
Proof. By drawing a diagram it is easily seen that if

$$
S<|(R+1) /(R-1)|,
$$

then $A_{R, S}=\{z=\mathrm{C} ;|z| \leqslant R,|(z-i) /(z+i)| \leqslant S\}$ is simply connected. Let $r>R, s>S$ oe such that

$$
A_{r, s}^{0}=\{z \in \mathbf{C} ;|z|<r,|(z-i) /(z+i)|<s\}
$$

is still simply connected. Then by Runge's theorem (Rudin [7, p. 258]) each holomorphic function in $A_{r, s}^{0}$ can be approximated, uniformly on compact sets, by polynomials. For every $m \geqslant 0, \int_{A_{R, S}} z^{m} d \mu(z)=f(m, 0)$ is known and, hence, $d \mu$ is determined on the polynomials. Since $A_{R, S}$ is a compact subset of $A_{r, s}^{0}, d \mu$ is uniquely determined by its restriction to the polynomials and, hence, $f$ is uniquely determined by $\{f(m, 0)\}_{0}^{\infty}$, the theorem is proved.

3. Discrete entire functions of exponential growth. In this section we deal with discrete entire functions, that is functions $f: Z \times Z \rightarrow C$ such that $L f=0$ on $Z \times Z$.

Let $Q$ be the algebra generated by $z, z^{-1}, w, w^{-1}$ :

$$
\mathcal{Q}=\left\{\sum_{-M}^{M} \sum_{-N}^{N} a_{m n} z^{m} w^{n} ; M, N \text { integers, } a_{m n} \in \mathrm{C}\right\} ;
$$

then as already mentioned in $\$ 1$, each discrete function $f: Z \times Z \rightarrow C$ induces a linear functional $T_{f}$ on

$$
T_{f}\left(\sum a_{m n} z^{m} w^{n}\right)=\sum a_{m n} f(m, n),
$$

Moreover, if $T$ is a linear functional on $\mathbb{Q}$ and $f(m, n)=T\left(z^{m} w^{n}\right)$, then $T=T_{f}$.

It follows much as in $\$ 2$ that $f(m, n)$ is discrete entire iff $T_{f}$ annihilates the ideal $(1+i z-z w-i w)$.

We state

Definition. A discrete function $f: Z \times Z \rightarrow \mathbf{C}$ is of exponential growth $(R, S)$ if there exists a constant $C$ such that

$$
|f(m, n)| \leqslant C R^{|m|} S^{|n|}
$$

for every $(m, n) \in Z \times Z$.

Let $R>1, S>1$ and $U_{R S}=\{1 / R<|z|<R\} \times\{1 / S<|w|<S\}$. The class of functions continuous on $\bar{U}_{R S}$ and holomorphic in $U_{R S}$ is a reflexive Banach space with sup norm which we shall denote by $\tilde{H}(R, S)$, and instead of Lemma (2.1) we have 
LEMMA 3.1. If $f(m, n)$ is of exponential growth $(R, S)$ and $r>R, s>S$, then $T_{f}$ defined on $\mathbb{Q}$ by (3.1) can be extended to be a continuous linear functional on the Banach space $\hat{H}(r, s)$.

There is an analogue to Theorem 2.2 also, which can be proved in much the same way.

THEOREM 3.2. Let $f(m, n)$ be discrete entire and of exponential growth $(R, S)(R>1, S>1)$. Then there exists a plane measure $d \mu(z)$ supported in

$$
\tilde{A}_{R, S}=\{z \in \mathrm{C} ; 1 / R \leqslant|z| \leqslant R, 1 / S \leqslant|(z-i) /(z+i)| \leqslant S\}
$$

such that

$$
f(m, n)=\int_{\tilde{A}_{R, S}} e(z ; m+i n) d \mu(z)
$$

for every $(m, n) \in Z \times Z$.

The measure $d \mu(z)$ in the above theorem is a continuous linear functional on the algebra of bounded holomorphic functions on the region $A_{r, s}^{0}$. At this point $(1)$ the following theorem due to Havin [8] is useful.

TheORem (HAvin). Let $G$ be an open set in $C$ and let $O(G)$ be the space of analytic functions on $G$. Put $F=C \sim G$ and assume $\infty \in F$. Then for every continuous linear functional $\phi$ on $O(G)$ there exists a unique locally analytic function $g_{\phi}$ such that if $g_{k}$ is analytic on some $G_{k} \supset F$ such that $\left.g_{k}\right|_{F}=g$, then

$$
\phi(f)=\frac{1}{2 \pi i} \int_{\Gamma} f(z) g_{k}(z) d z
$$

where $\Gamma$ is a contour in $G \cap G_{k}$.

Applying this theorem to our functional $d \mu(z)$ on the space of bounded holomorphic functions on the region $\tilde{A}_{r, s}$ we have

THEOREM 3.3. Let $f(m, n)$ be discrete entire and of exponential growth $(R, S)$; let $r>R, s>S$. Put $\tilde{B}_{r s}=\mathbf{C}^{2} \sim \tilde{A}_{r s}^{0}$; then there exists a unique locally analytic function $g$ such that if $g_{k}$ is analytic on some $G_{k} \supset B_{r, s}$ such that $\left.g_{k}\right|_{B_{r}}=g$, then

$$
f(m, n)=\frac{1}{2 \pi i} \int_{\Gamma} e(z ; m+i n) g_{k}(z) d z
$$

where $\Gamma \subset A_{r, s}^{0} \cap G_{k}$.

(') The impatient reader may skip immediately to the Paley-Wiener theorem (Theorem 3.4), the proof of which is independent of the present circle of ideas. 
The above theorem can be viewed as the discrete analogue of the representation theorem for entire functions of exponential type (cf. Boas [3, p. 74]): "If $f(z)$ is an entire function of exponential type, $D$ is its conjugate indicator diagram and $C$ is a contour containing $D$ in its interior, then

$$
f(z)=\frac{1}{2 \pi i} \int_{C} F(\omega) e^{z \omega} d \omega
$$

where $F(\omega)$ is the so-called Borel transform of $f(z)$."

Imitating continuous usage, we may call the support of $d \mu$ the "conjugate indicator diagram" and the function $g$ of Theorem 3.3 the "Borel transform". Notice that the support of $d \mu$ (the "conjugate indicator diagram") is not, in general, simply connected.

We shall finish this paper with a discrete analogue to the celebrated twosided Paley-Wiener theorem (Boas [3, p. 103]):

Theorem (PAley-Wiener). The entire function $f(z)$ is of exponential type $\tau$ and belongs to $L^{2}$ on the real axis iff

$$
f(z)=\int_{-\tau}^{\tau} e^{i z t} \phi(t) d t
$$

where $\phi(t) \in L^{2}(-\tau, \tau)$.

In the following $T$ will denote the unit circle $\{|z|=1\}$.

THEOREM 3.4. Let $f(m, n)$ be discrete entire and of exponential growth $(R, S)$ where $S<|(R+1) /(R-1)|$ and suppose it belongs to $L^{2}$ on the discrete real line $\sum_{m=-\infty}^{\infty}|f(m, 0)|^{2}<\infty$; then there exists a function $\phi \in L^{2}(T)$ whose support is a compact subset of $T \sim\{i,-i\}=\{z \in \mathbf{C} ;|z|=1, z \neq \pm i\}$ such that

$$
\begin{aligned}
f(m, n) & =\frac{1}{2 \pi} \int_{T} \phi(z) e(z ; m+i n) d z \\
& =\frac{1}{2 \pi} \int_{-\pi}^{\pi}\left[i \phi\left(e^{i t}\right) e^{i t}\right] e^{i m t}\left(\frac{1+i e^{i t}}{e^{i t}+i}\right)^{n} d t .
\end{aligned}
$$

Proof. By Theorem 3.2, $f(m, n)=\int e(z ; m+i n) d \mu(z)$ for some measure $d \mu$ supported in $\tilde{A}_{R, S}=\{z \in \mathrm{C} ; 1 / R \leqslant|z| \leqslant R, 1 / S \leqslant|(z-i) /(z+i)|<S\}$. Since $S<|(R+1) /(R-1)|$ the complement of $\tilde{A}_{R, S}$ is connected $\left(\tilde{A}_{R, S}\right.$ consists of two simply connected components, one containing $z=1$ and the other $z=-1$ ).

Let $r>R, s>S$ be sufficiently close to $R, S$ (respectively) to make the complement of $\tilde{A}_{r, s}^{0}=\{z \in \mathrm{C} ; 1 / r<|z|<r, 1 / s<|(z-i) /(z+i)|<s\}$ connected. Then by Runge's theorem (Rudin [7, p. 258]) every bounded 
holomorphic function on $\overleftarrow{A}_{r, s}^{0}$ can be approximated uniformly on compact sets by polynomials. It follows that the values $\int z^{m} d \mu(z), m=0, \pm 1, \pm 2, \ldots$, determine $d \mu$. Also for every polynomial $u(z)=\sum_{-M}^{M} a_{m} z^{m}$,

$$
\int u(z) d \mu(z)=\sum_{-M}^{M} a_{m} f(m, 0) .
$$

Since $\sum_{-\infty}^{\infty}|f(m, 0)|^{2}<\infty, d \mu$ can be extended to be a linear functional on $L^{2}\left(T \cap \tilde{A}_{R, S}\right)$, and by Riesz' representation theorem there exists a function $\phi(z) \in L^{2}\left(T_{R S}\right)$ (where $T_{R S}=T \cap \tilde{A}_{R, S}$ ), such that for every bounded holomorphic function $u(z)$ on $\tilde{A}_{r, s}^{0}$ (which automatically then belongs to $L^{2}\left(T_{R S}\right)$ ):

$$
\int_{\tilde{A}_{R, S}} u(z) d \mu(z)=\frac{1}{2 \pi} \int_{T_{R S}} \phi(z) u(z) d z
$$

In particular,

$$
f(m, n)=\frac{1}{2 \pi} \int_{T_{R, S}} e(z ; m+i n) \phi(z) d z .
$$

$T_{R S}=T \cap \tilde{A}_{R, S}$ is a compact subset of $T \sim\{i,-i\}$ and, evidently,

$$
i e^{i t} \phi\left(e^{i t}\right)=\sum_{-\infty}^{\infty} f(m, 0) e^{-i m t}
$$

vanishes, a.e., outside $T_{R S}$.

ACKNowledgement. The author wishes to thank Dr. Shmuel Kiro and Professor Harry Dym for helpful comments and discussions.

\section{REFERENCES}

1. Jacqueline Ferrand, Fonctions préharmoniques et fonctions préholomorphes, Bull. Sci. Math. (2) 68 (1944), 152-180. MR 7, 149.

2. R. J. Duffin, Basic properties of discrete analytic functions, Duke Math. J. 23 (1956), 335-363. MR 17, 1193.

3. R. P. Boas, Jr., Entire functions, Academic Press, New York, 1954. MR 16, 914.

4. L. Ehrenpreis, Fourier analysis in several complex variables, Interscience, New York and London, 1970. MR 44 \#3066.

5. W. Rudin, Function theory in polydiscs, Benjamin, New York, 1969. MR 41 \#501.

6. A. E. Taylor, Introduction to functional analysis, Wiley, New York, 1958. MR 20 \#5411.

7. W. Rudin, Real and complex analysis, McGraw-Hill, New York, 1966. MR 35 \# 1420.

8. V. P. Havin, Spaces of analytic functions, Math. Analysis 1964, Akad. Nauk SSSR Inst. Naužn. Informacii, Moscow, 1966, pp. 76-164. (Russian) MR 34 \#6512.

9. R. C. Gunning and H. Rossi, Analytic functions of several complex variables, Prentice-Hall, Englewood Cliffs, N.J., 1965. MR 31 \#4927.

Department of Mathematics, Weizmann Institute of Science, Rehovot, Isruel 